\title{
Histopathological evaluation including cytokeratin 13 and Ki-67 in the border between Lugol-stained and -unstained areas
}

\author{
KOUJI OHTA ${ }^{1}$, IKUKO OGAWA $^{3}$, SHIGEHIRO ONO $^{1}$, MASAAKI TAKI $^{1}$, KUNIKO MIZUTA $^{1}$, \\ MIWA MIYAUCHI ${ }^{1}$, MASAAKI TAKECHI ${ }^{1}$, HIDEO SHIGEISHI ${ }^{1}$, TAKASHI TAKATA $^{2}$ and NOBUYUKI KAMATA ${ }^{1}$ \\ ${ }^{1}$ Department of Oral and Maxillofacial Surgery, Division of Cervico-Gnathostomatology, Programs for Applied Biomedicine and \\ ${ }^{2}$ Department of Oral and Maxillofacial Pathobiology, Division of Frontier Medical Science, Graduate School of Biomedical \\ Sciences, Hiroshima University; ${ }^{3}$ Center of Oral Clinical Examination, Hiroshima University Hospital, Hiroshima, Japan
}

Received November 11, 2009; Accepted January 8, 2010

DOI: $10.3892 /$ or_00000822

\begin{abstract}
Lugol's iodine staining (Lugol-staining) has been widely used to detect malignant changes in the cervix uteri and esophagus. However, pathological and histochemical changes in the border between Lugol-stained and -unstained areas in oral epithelial dysplastia and malignant lesions are not well understood. We examined the histological appearance of 20 cases of epithelial dysplasia surrounding squamous cell carcinoma using HE and PAS staining in the borders between Lugol-stained and -unstained areas. Subsequently, differences in the expression of cytokeratin 13 (CK13), an epithelium differentiation marker, and Ki-67, a cell proliferative marker, in those areas were investigated by immunohistochemistry. Lugol-stained areas of all cases showed mild dysplasia or normal epithelium appearance, while Lugol-unstained areas in most cases were diagnosed as moderate/severe dysplasia and carcinoma in situ. PAS reactions were limited or not found in the Lugol-unstained areas as compared to intense positivity in Lugol-stained areas. CK13 and Ki-67 protein expression was significantly different between Lugol-stained and -unstained areas. It was confirmed that epithelia showing precancerous or cancerous features were detected as Lugol-unstained boundary areas. A reduction in glycogen production caused by alterations of cell differentiation and proliferation associated with malignant changes may result in a lack of Lugol-staining.
\end{abstract}

\section{Introduction}

Dysplastic changes, have a high potential for malignant transformation and, can be found in epithelium surrounding an oral squamous cell carcinoma $(1,2)$. However, the region of

Correspondence to: Dr Kouji Ohta, Division of CervicoGnathostomatology, Department of Oral and Maxillofacial Surgery, Programs for Applied Biomedicine, Graduate School of Biomedical Sciences, Hiroshima University, 1-2-3 Kasumi, Minami-Ku, Hiroshima 734-8553, Japan

E-mail: otkouji@hiroshima-u.ac.jp

Key words: Lugol's iodine staining, cytokeratin 13, Ki-67 epithelial dysplasia is not be easily distinguished macroscopically from normal epithelium, thus Lugol's iodine staining (Lugol-staining) is widely used to detect malignant changes in the cervix uteri and esophagus $(3,4)$. On the other hand, few studies have demonstrated the utility of Lugolstaining for detecting oral malignant lesions, and it has not been clarified whether Lugol-unstained areas surrounding oral dysplasia and a squamous cell carcinoma should be categorized as pre-malignant epithelia and resected.

Lugol-staining is linked to a reaction with glycogen in the cytoplasm (5). Malignant cervical lesions have a limited amount of glycogen as compared with normal squamous epithelium, thus they are revealed by a lack of Lugolstaining, whereas normal areas are dyed a brown-black color (6). However, changes related to cell differentiation and proliferation in the borders between Lugol-stained and -unstained areas in cases with oral dysplastic lesions are not well understood.

Keratins are considered to be excellent markers of keratinocyte differentiation and can reflect different patterns of keratinization. The expression of specific types, such as cytokeratin (CK)4 and CK13, is decreased in dysplastic lesions in the oral epithelium, and such changes have been associated with the development of oral dysplasia and squamous cell carcinomas $(7,8)$. On the other hand, Ki-67 is a representative molecular marker of proliferating cells, and its labeling index correlates with the grade of dysplasia and severity of malignancy $(9,10)$. In the present study, to elucidate the changes related to cell differentiation and proliferation in borders distinguished by Lugol-staining in and epithelial dysplasia surrounding squamous cell carcinoma, we investigated the histological appearance in the borders between Lugol-stained and -unstained areas using hematoxylin and eosin (HE) and periodic acid-Sciff (PAS)staining. Subsequently, we examined the changes in CK13 and $\mathrm{Ki}-67$ protein expression in those areas using immunohistochemistry.

\section{Materials and methods}

Twenty cases of oral epithelial dysplasia surrounding oral dysplasia and oral squamous cell carcinoma arising from 


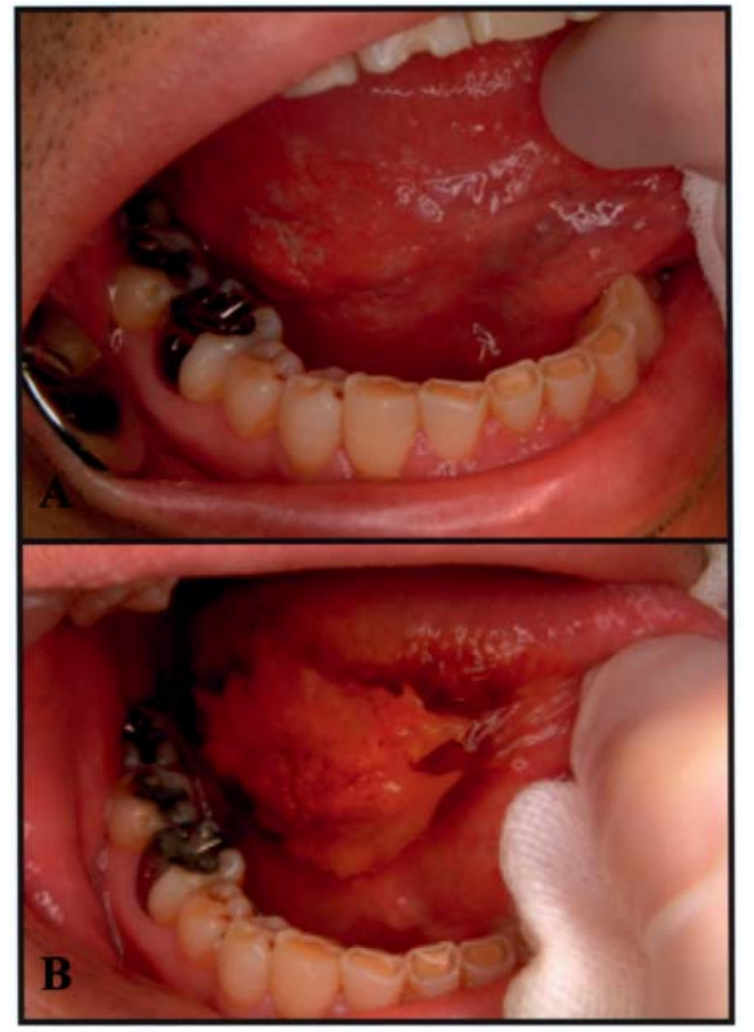

Figure 1. Oral epithelial dysplasia surrounding squamous cell carcinoma of the tongue. (A) Clinical appearance before Lugol-staining. (B) Appearance after Lugol-staining. mucosa of the tongue, floor of the mouth or buccal mucosa, which were made up of a weakly parakeratinized epithelium, were examined in this study. Because a highly keratinized epithelium does not normally retain the Lugol solution. Lugol-staining was performed as follows. First, the lesion was rinsed with water and dried, then, it and surrounding area were subjected to Lugol-staining using a cotton swab before resection (Fig. 1). About 2 min after application, specimens from the boundary area between Lugol-stained and -unstained areas were obtained from $5 \mathrm{~mm}$ outside the boundary line, marked by an incision made along the boundary. Informed consent for the procedure was obtained from each patient according to a protocol approved by the Ethics Committee of Hiroshima University.

Light microscopy. The specimens were fixed in $10 \%$ formalin and embedded in paraffin, then sections $\sim 5 \mu \mathrm{m}$ thick were cut and stained with HE and PAS. Two investigators confirmed the diagnosis of the specimens according to criteria proposed by the WHO.

Immunohistochemistry. Immunohistochemical staining of formalin-fixed, paraffin-embedded sections was done using an Envision kit (Dako, Glostrup, Denmark). After blocking endogenous peroxidase with methanol containing $0.3 \%$ $\mathrm{H}_{2} \mathrm{O}_{2}$ for $30 \mathrm{~min}$, microwave pretreatment in citrate buffer ( $\mathrm{pH}$ 6.0) was performed for antigen retrieval. The sections were incubated with the primary antibodies for CK13 and

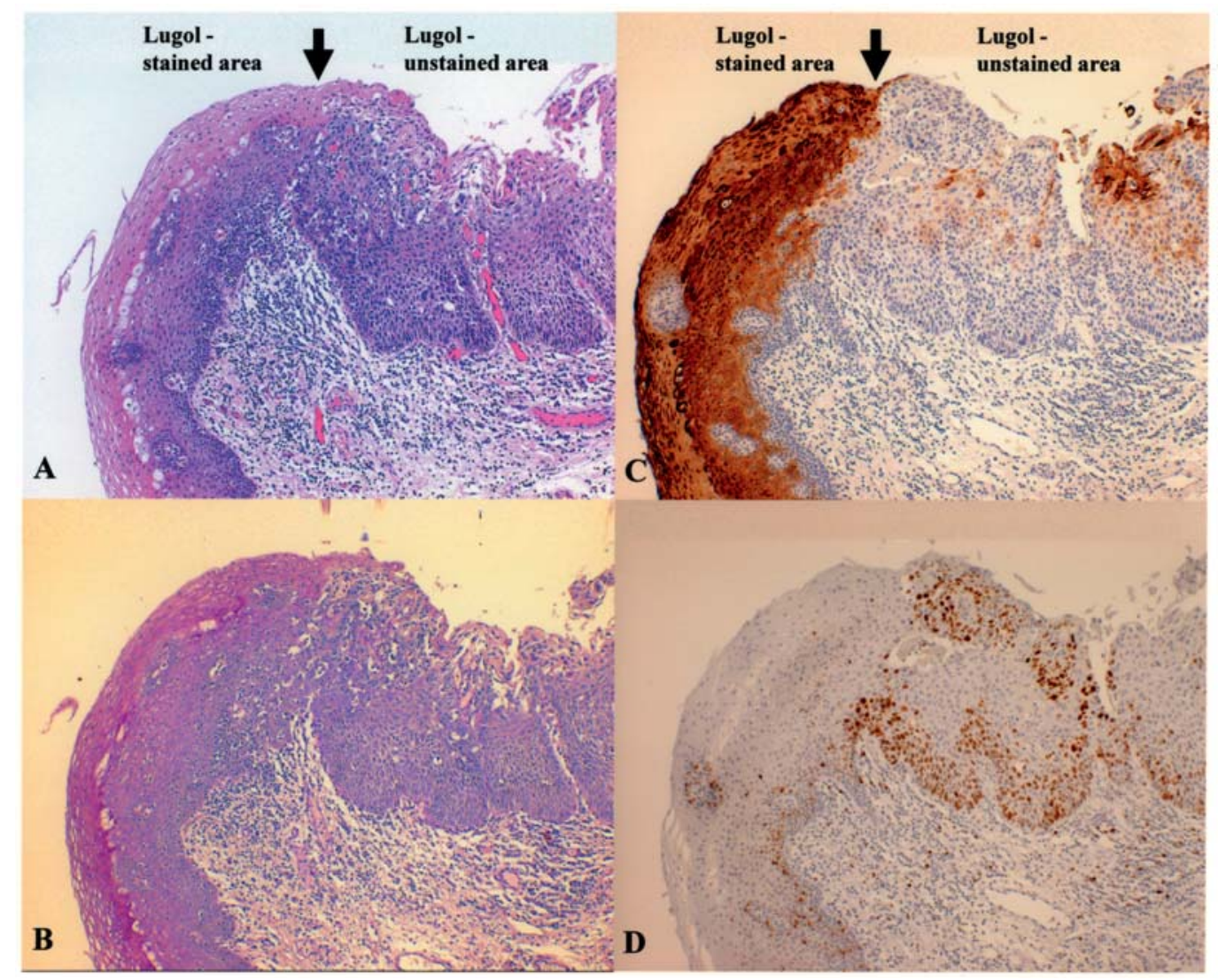

Figure 2. Histopathological and immunohistological findings in the border between Lugol-stained and -unstained areas in case 1. (A) HE staining, (B) PAS staining (C), CK13 immunostaining, (D) Ki-67 immunostaining. Small arrows indicate the border between Lugol-stained and unstained areas. 
Table I. Histological diagnosis in the border between Lugol-stained and -unstained areas.

\begin{tabular}{|c|c|c|c|c|}
\hline \multirow[b]{2}{*}{ Case no. } & \multirow[b]{2}{*}{ Gender/Age } & \multirow[b]{2}{*}{ Location } & \multicolumn{2}{|c|}{ Histological diagnosis ${ }^{\mathrm{a}}$} \\
\hline & & & Lugol-stained area & Lugol-unstained area \\
\hline 1 & $\mathrm{M} / 59$ & Tongue & Mild & Severe/CIS \\
\hline 2 & $\mathrm{~F} / 77$ & Tongue & Normal, mild & Moderate, severe/CIS \\
\hline 3 & $\mathrm{~F} / 68$ & Tongue & Normal, mild & Moderate \\
\hline 4 & $\mathrm{M} / 66$ & Tongue & Mild & Severe/CIS \\
\hline 5 & $\mathrm{~F} / 80$ & Buccal mucosa & Normal, mild & Severe/CIS \\
\hline 6 & $\mathrm{M} / 83$ & Buccal mucosa & Normal & Moderate \\
\hline 7 & $\mathrm{M} / 56$ & Tongue & Normal & Moderate \\
\hline 8 & $\mathrm{~F} / 80$ & Tongue & Normal, mild & Severe/CIS \\
\hline 9 & $\mathrm{~F} / 73$ & Tongue & Normal & Mild, moderate \\
\hline 10 & $\mathrm{M} / 68$ & Tongue & Normal & Severe/CIS \\
\hline 11 & $\mathrm{M} / 74$ & Tongue & Normal & Mild, moderate \\
\hline 12 & $\mathrm{M} / 70$ & Mouth floor & Normal & Moderate \\
\hline 13 & $\mathrm{M} / 69$ & Tongue & Normal & Mild, moderate \\
\hline 14 & $\mathrm{M} / 44$ & Tongue & Normal & Moderate \\
\hline 15 & $\mathrm{M} / 58$ & Tongue & Mild & Severe/CIS \\
\hline 16 & $\mathrm{~F} / 43$ & Tongue & Normal, mild & Moderate, severe/CIS \\
\hline 17 & $\mathrm{M} / 56$ & Tongue & Normal & Moderate, severe/CIS \\
\hline 18 & $\mathrm{M} / 65$ & Tongue & Mild & Severe/CIS \\
\hline 19 & $\mathrm{~F} / 72$ & Tongue & Normal, mild & Moderate, severe/CIS \\
\hline 20 & $\mathrm{M} / 35$ & Tongue & Normal & Mild, moderate \\
\hline
\end{tabular}

${ }^{a}$ Mild, moderate and severe indicate the degree of dysplasia. CIS indicates carcinoma in situ.

Ki-67 (Dako, working dilutions $1 / 100$ and 1/50, respectively) overnight at $4^{\circ} \mathrm{C}$, followed by incubation with labeled polymer-peroxidase anti-mouse immunoglobulins for $30 \mathrm{~min}$ at room temperature. Color was developed with 3,3'-diaminobenzidine. Specificity was ascertained by substituting normal mouse IgG for the primary antibody. Two investigators evaluated the intensity of staining for CK13, which was classified as non-staining or sporadic (-), weak and/or patchy $(+)$, strong and homogeneous (++). Ki-67 labeling index was determined by the examination of at least 500 epithelial cells at x200 magnification in Lugol-stained or -unstained areas of each case and classified as $0-5 \%(+), 6-25 \%(++)$, $>25 \%(+++)$.

\section{Results}

Tissue samples from the border between Lugol-stained and - unstained areas contained mucous epithelia with various histopathological findings, including no obvious changes, various degrees of dysplasia, and carcinoma in situ. In all the cases stained by Lugol solution in the border areas, the mucous epithelium showed normal appearance or only slightly dysplastic changes (Table I). On the other hand, Lugol-unstained areas were histopathologically diagnosed as moderate to severe dysplasia or carcinoma in situ in 16 cases (Table I). In 12 of those cases, the demarcation between normal or mild dysplastic and highly dysplastic epithelium including carcinoma in situ, was clear and easily identifiable in the HE-stained sections (Fig. 2A). In the other 4 cases, the degree of dysplasia gradually decreased and dysplastic changes such as abnormal variation in cell size, increased nuclear-cytoplasmic ratio, or increased nuclear size, spread to the basal/parabasal layers of the epithelium in the border area (Fig. 3A). In Lugol-stained areas, the surface of the epithelium showed regular parakeratosis, whereas Lugol-unstained areas were covered with highly or irregularly parakeratinized or orthokeratinized epithelium (Table II). Eight cases had an orthokeratinized layer in Lugol-unstained areas, of which 3 were consistent with cases histologically evaluated as having mild to moderate dysplasia in the border (Table II).

Lugol-stained areas corresponded with epithelia showing PAS-positive reactions. In these areas, a large number of PAS-positive glycogen granules were observed in the cytoplasm of epithelial cells on the surface and in prickle cell layers (Figs. 2B and 3B). In contrast, epithelial cells in Lugolunstained areas had limited or no PAS reactions (Figs. 2B and $3 \mathrm{~B}$ ). Although negativity for PAS-staining was generally related to a high grade of epithelial dysplasia, including carcinoma in situ, in the border of Lugol-staining, the dividing line between the PAS-positive and -negative areas did not necessarily correspond with the border line between normal or mild dysplasia and moderate to severe dysplasia/carcinoma in situ observed in HE-stained sections. In 9 cases, the 


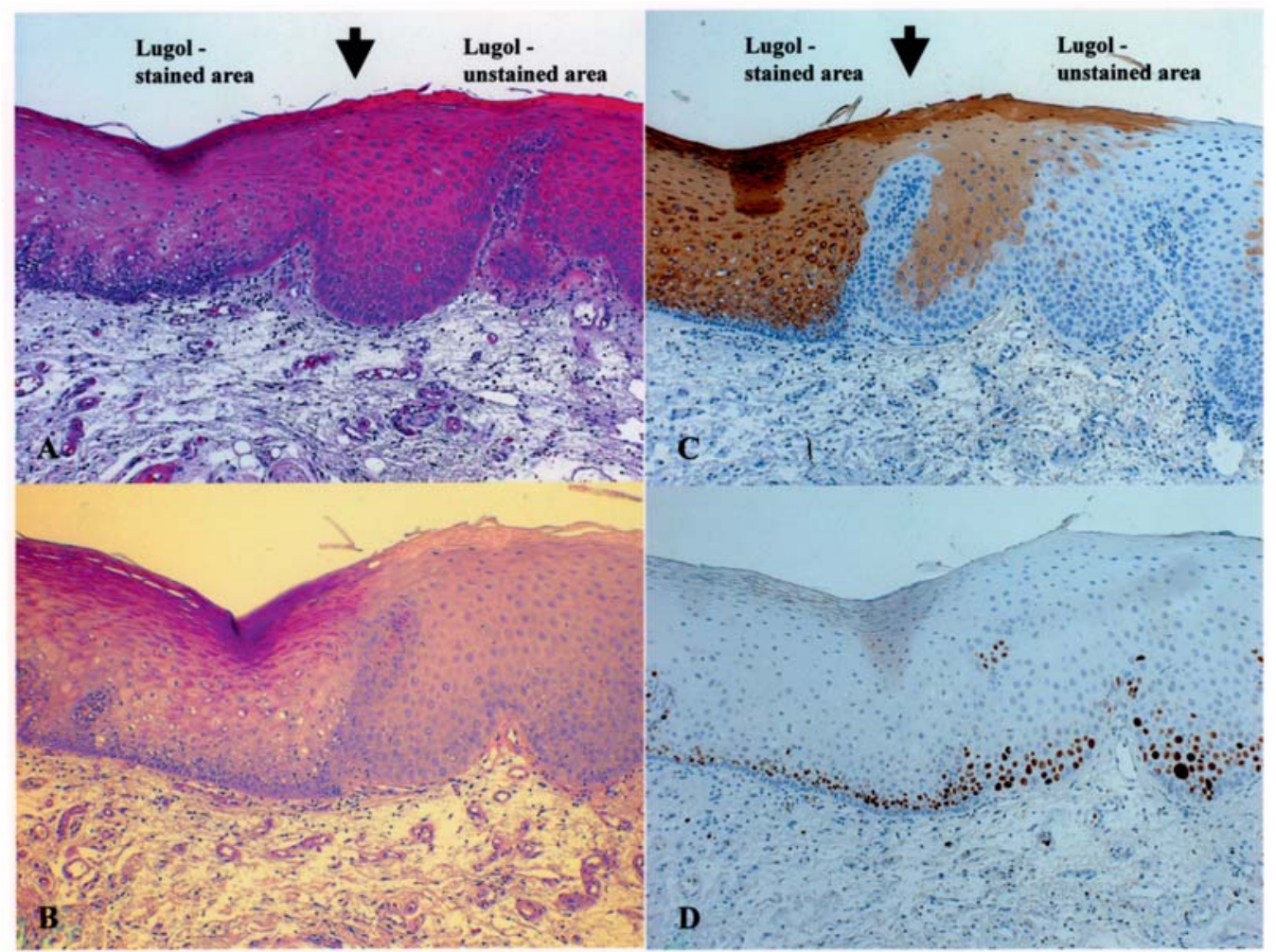

Figure 3. Histopathological and immunohistological findings in the border between Lugol-stained and -unstained areas in case 2. (A) HE staining, (B) PAS staining, (C) CK13 immunostaining (D) Ki-67 immunostaining. Small arrows indicate the border between Lugol-stained and -unstained areas.

Table II. Histological findings of the surface epithelium and expression of CK13 and Ki-67 protein in the border between Lugol-stained and -unstained areas.

\begin{tabular}{|c|c|c|c|c|c|c|}
\hline \multirow[b]{2}{*}{ Case no. } & \multicolumn{3}{|c|}{ Lugol-stained area } & \multicolumn{3}{|c|}{ Lugol-unstained area } \\
\hline & Keratinization $^{\mathrm{a}}$ & CK13 ${ }^{b}$ & $\mathrm{Ki}-67^{\mathrm{c}}$ & Keratinization $^{\mathrm{a}}$ & CK13 ${ }^{b}$ & $\mathrm{Ki}-67^{\mathrm{c}}$ \\
\hline 1 & PK & ++ & + & PK & + & +++ \\
\hline 2 & PK & ++ & + & PK/OK & - & ++ \\
\hline 3 & PK & ++ & + & OK & + & ++ \\
\hline 4 & PK & ++ & + & $\mathrm{PK} / \mathrm{OK}$ & - & ++ \\
\hline 5 & PK & ++ & + & PK & - & +++ \\
\hline 6 & PK & ++ & + & PK & + & ++ \\
\hline 7 & PK & ++ & + & PK & + & ++ \\
\hline 8 & PK & ++ & + & PK & + & +++ \\
\hline 9 & PK & ++ & + & OK & + & ++ \\
\hline 10 & PK & ++ & + & PK & + & +++ \\
\hline 11 & PK & ++ & + & PK & + & ++ \\
\hline 12 & PK & ++ & + & OK & + & ++ \\
\hline 13 & PK & ++ & + & $\mathrm{PK} / \mathrm{OK}$ & + & ++ \\
\hline 14 & PK & ++ & + & PK & + & ++ \\
\hline 15 & PK & ++ & + & PK & - & +++ \\
\hline 16 & PK & ++ & + & PK & + & ++ \\
\hline 17 & PK & ++ & + & PK & + & ++ \\
\hline 18 & PK & ++ & + & PK & - & +++ \\
\hline 19 & PK & ++ & + & $\mathrm{PK} / \mathrm{OK}$ & + & +++ \\
\hline 20 & PK & ++ & + & $\mathrm{OK}$ & + & ++ \\
\hline
\end{tabular}

a PK indicates parakeratosis. OK indicates orthokeratosis. ${ }^{\mathrm{b}} \mathrm{CK} 13$ expression is indicated as non-staining or sporadic (-), weak and/or patchy $(+)$, and strong and homogeneous $(++)$. ${ }^{c} \mathrm{Ki}-67$ expression is indicated as $0-5 \%(+), 6-25 \%(++)$, and $>25 \%(+++)$. 
boundary line of PAS-staining was identical to that of the pathological border evaluated in HE-stained sections, whereas it was located on the lesional side of the pathological border in 11. In those latter cases, a large number of PAS-positive granules was also present on the surface of the dysplastic epithelia. In addition, orthokeratinized areas were always negative for PAS-staining regardless of the degree of epithelial dysplasia.

CK13 and Ki-67 expressions were significantly different between Lugol-stained and -unstained areas (Table II). CK13 was regularly and highly expressed throughout the epithelium except for basal cells in Lugol-stained areas (Figs. 2C and $3 \mathrm{C})$. Whereas, that expression was markedly reduced in Lugol-unstained areas, and sporadically or weakly expressed in the border (Figs. 2C and 3C). Ki-67 was detected in basal/parabasal cells of Lugol-stained areas, though it was more widely expressed in unstained areas (Figs. 2D and $3 \mathrm{D})$. The changes in expressions of these two proteins were related to the pathological border observed in HE-stained sections.

\section{Discussion}

In the present study, we found that alterations of CK13 and $\mathrm{Ki}-67$ protein expression between Lugol-stained and -unstained areas of oral dysplasia surrounding squamous cell carcinoma. CK13 is a differentiation-specific keratin, and Bloor et al reported that its protein expression was reduced in moderate and severe dysplasia (7). Furthermore, the level of CK13 mRNA expression in squamous cell carcinoma and severe dysplasia was reported to be lower than that in mild to moderate dysplasia (8). The present results showed that CK13 protein was intensely and regularly expressed in epithelia, except for basal cells in Lugol-stained areas, whereas it was markedly decreased in Lugol-unstained areas. The differences in expression clearly demonstrated the boundary line, which was consistent with the pathological border in HE-stained sections. Ki-67 protein is a representative molecular marker of proliferating cells that is extensively used in examination of oral epithelial dysplasia and squamous cell carcinoma $(9,10)$. In the present study, Ki-67 protein expression was detected in the basal/parabasal layers of Lugol-stained areas, whereas Ki-67 positive cells were increased in Lugol-unstained areas and also found in suprabasal prickle cell layers. In the border between Lugol-stained and -unstained areas, differences in the number and localization of Ki-67-positive cells were observed. These findings correspond with previous findings showing that the total number of proliferating cells, which increases according to the degree of epithelial dysplasia and aggregation of $\mathrm{Ki}-67$-positive cells in the suprabasal layer, is a useful marker of high-grade dysplasia (11). Our results obtained with the immunohistochemical staining for CK13 and Ki-67 confirmed the ability of Lugol-staining to demonstrate the precise border between low- and high-grade dysplasia. Differentiation and proliferation changes related to malignant transformation may occur in dysplastic epithelium in the border between Lugol-stained and -unstained areas.

Lugol-staining produces a brown-black color based on the reaction of iodine with glycogen granules (12). The tongue, cheek and mouth floor areas are covered with slightly parakeratotic epithelium that contains glycogen granules in the cytoplasm of the prickle and surface cell layers, resulting in positivity for Lugol-staining. Glycogen content is inversely related to the degree of keratosis, suggesting a role of glycogen in keratinization (13). Malignant epithelium has a limited amount of glycogen granules as compared with normal squamous epithelium (3). It has been reported that intermediate filaments, precursors of keratosis, in epithelial cells are increased in the cytoplasm according to the degree of epithelial dysplasia, while glycogen granules in moderate and severe dysplasia are significantly decreased compared with those in the normal epithelium (14). In the present study, all border areas stained by Lugol solution were normal or mild dysplastic epithelium corresponded with PAS-positive, which indicates parakeratotic epithelium containing a large amount of glycogen. On the other hand, Lugo-unstained areas, which were PAS-negative, showed irregularly parakeratotic or orthokeratotic epithelium, in which the degree of dysplasia was higher and the amount of glycogen lower as compared to Lugol-stained areas. However, the dividing lines between the PAS-positive and -negative areas were not necessarily in agreement with the pathological borders. Kurita et al reported that wide differences were occasionally found between the dividing lines in dysplastic epithelia denoted by PAS staining and those by iodine staining, and noted that the branched structure of glycogen granules may break down in the process of degeneration, while the PAS-reactive character remained (15). Thus, glycogen granules in the border of the Lugol-unstained area may be in the process of degeneration. The correlation between CK13 expression and glycogen content suggests that loss of cellular differentiation may render the cells incapable of producing glycogen (16). Therefore, a reduction in glycogen production caused by alterations of cell differentiation and proliferation associated with malignant changes may result in the lack of Lugol-staining.

Some investigators examined whether the Lugol-unstained region surrounding an oral squamous cell carcinoma should be resected surgically together with the tumor. Yajima et al reported that telomerase activity was significantly different between iodine-stained areas of normal epithelium and -unstained areas of epithelial dysplasia (17). In another study, p53 expression in iodine-unstained areas was markedly higher than in iodine-stained areas (14). Our findings along with those suggest that the Lugol-unstained area is consistent with premalignant and malignant lesions in the oral mucosa, and should be completely removed if it surrounds dysplasia or a squamous cell carcinoma.

In our study, Lugol-staining was helpful to determine surgical resection margins for removal of oral dysplastic epithelium. However, application is limited to non-keratinized mucosa, such as that of the tongue, cheek and oral floor, while this method also can not detect subepithelial infiltrating tumors (15). Based on our results, slight dysplasia categorized as mild grade could not be distinguished from normal epithelium by Lugol-staining. Additional clinical studies and long-time follow-up results are needed to distinguish surgical resection margins from Lugol-unstained areas in oral epithelial dysplasia surrounding a squamous cell carcinoma. 


\section{References}

1. Gluckman JL, Crissman JD and Donegan JO: Multicentric squamous-cell carcinoma of the upper aerodigestive tract. Head Neck Surg 3: 90-96, 1980.

2. Eliezri YD, Israel HA and Pochal WF: Treatment of an oral erythroplastic squamous cell carcinoma with Mohs' micrographic surgery. Oral Surg Oral Med Oral Pathol 67: 249-254, 1989.

3. Richart RM: A clinical staining test for the in vivo delineation of dysplasia and carcinoma in situ. Am J Obstet Gynecol 86: 703-712, 1963

4. Sugimachi K, Kitamura K, Baba K, Ikebe M and Kuwano H: Endoscopic diagnosis of early carcinoma of the esophagus using Lugol's solution. Gastrointest Endosc 38: 657-661, 1992.

5. Akasaka Y, Okuda J, Ida K, Toriie S, Nishino H and Kimoto K: Vital staining of esophageal mucosa using endoscopic spraying technique of Lugol's solution-for the aid of esophagoscopic diagnosis of the cancer. Gastoroenterol Endosc 18: 84-92, 1976.

6. Shedd DP, Hukill $\mathrm{P}$ and Bahn S: In vivo staning properties of oral cancer. Am J Surg 110: 631-634, 1965.

7. Bloor BK, Seddon SV and Morgan PR: Gene expression of differentiation-specific keratins in oral epithelial dysplasia and squamous cell carcinoma. Oral Oncol 37: 251-261, 2001.

8. Ohkura S, Kondoh N, Hada A, Arai M, Yamazaki Y, Sindoh M and Takahashi M, Matsumoto I and Yamamoto M: Differential expression of the keratin-4, $-13,-14,-17$ and transglutaminase 3 genes during the development of oral squamous cell carcinoma from leukoplakia. Oral Oncol 41: 607-613, 2005.

9. Kushner J, Bradley G and Jordan RC: Patterns of p53 and Ki-67 protein expression in epithelial dysplasia from the floor of the mouth. J Pathol 183: 418-423, 1997.
10. Saito T, Nakajima T and Mogi K: Immunohistochemical analysis of cell cycle-associated proteins p16, pRb, p53, p27 and Ki-67 in oral cancer and precancer with special reference to verrucous carcinomas. J Oral Pathol Med 28: 226-232, 1999.

11. Takeda T, Sugihara K, Hirayama Y Hirano M, Tanuma JI and Semba I: Immunohistological evaluation of Ki-67, p63, CK19 and p53 expression in oral epithelial dysplasias. J Oral Pathol Med 35: 369-375, 2006.

12. Epstein JB, Scully C and Spinelli J: Toluidine blue and Lugol's iodine application in the assessment of oral malignant disease and lesions at risk of malignancy. J Oral Pathol Med 21: 160-163, 1992.

13. Silverman S Jr, Barbosa J and Keams G: Ultrastructural and histochemical localization of glycogen in human normal and hyperkeratotic oral epithelium. Arch Oral Biol 16: 423-434, 1971.

14. Yokoo K, Noma H, Inoue $\mathrm{T}$, Hashimoto $\mathrm{S}$ and Shimono M: Cell proliferation and tumour suppressor gene expression in iodine unstained area surrounding oral squamous cell carcinoma. Int J Oral Maxillofac Surg 33: 75-83, 2004.

15. Kurita $\mathrm{H}$ and Kurashina $\mathrm{K}$ : Vital staining with iodine solution in delineating the border of oral dysplastic lesions. Oral Surg Oral Med Oral Pathol Oral Radiol Endod 81: 275-280, 1996.

16. Doyle JL, Manhold JH and Weisinger E: Study of glycogen content and basement membrane in benign and malignant oral lesions. Oral Surg Oral Med Oral Pathol Oral Radiol Endod 26: 667-673, 1968.

17. Yajima Y, Noma H, Furuya Y Nomura T, Yamauchi T, Kasahara K, Hatada K and Takano M: Quantification of telomerase activity of regions unstained with iodine solution that surround oral squamous cell carcinoma. Oral Oncol 40: 314-320, 2004 